\title{
Lapurdum
}

Euskal ikerketen aldizkaria | Revue d'études basques |

Revista de estudios vascos | Basque studies review

2| 1997

Numéro II

\section{Hiru aditz aurrizki zahar 16. mendeko testuetan.}

A propos de trois anciens préfixes verbaux dans les textes $d u 16^{e}$ siècle

\section{Bernard Oyharçabal}

\section{OpenEdition \\ Journals}

Édition électronique

URL : http://journals.openedition.org/lapurdum/1784

DOI : 10.4000/lapurdum.1784

ISSN : 1965-0655

Éditeur

IKER

Édition imprimée

Date de publication : 1 octobre 1997

Pagination : 45-62

ISBN : 2-84127-142-0

ISSN : $1273-3830$

Référence électronique

Bernard Oyharçabal, « Hiru aditz aurrizki zahar 16. mendeko testuetan. », Lapurdum [Linean], 2 | 1997

Sarean emana----an 01 septembre 2010, kontsultatu 01 février 2020. URL : http:// journals.openedition.org/lapurdum/1784; DOI : 10.4000/lapurdum. 1784 


\section{HIRU ADITZ AURRIZKI ZAHAR 16. MENDEKO TESTUETAN.}

Adizkera jokatuetan agertzen diren elementuen artean, luzaz, euskal gramatikariek ez dituzte elkarretarik berezi, aditz jokadurari berari dagozkion markak eta menderakuntzari dagozkionak. Hobeki errateko, aditzaren beraren jokadurako flexio gisa azterkatu izan dituzte menderagailu batekin agertzen diren adizkerak, morfologiaren eta joskeraren arteko aldea kontuan hartu gabe. Ikuspegi horten ondorio adierazgarrienetarik bat da Humboltek (1817) euskal partizipioez erran zuena, harentzat perpaus erlatibo libroen adizkerak (dakidana) baitziren egiazko partizipioak, ideia bera errepikatzen zuelarik haren ondotik A. d'Abbadiek (1836) ere.

Perpaus jokatuetan menderagailu gisa baliatzen ditugun morfemek bi ezaugarri dituzte : lehenik, orain gogorarazi dugun bezala, adizkera jokatuari lotzen zaizkio, harekin morfologian bat egiten dutelarik ; bigarrenekorik, hizki gisa agertzen dira : edo atzizki gisa. edo aurrizki gisa.

Euskara tipologikoki hizkuntza posposizionala denaz geroz, ez da harrigarria atzizki gisa agertzea menderagailuak. Are gehiago, azterketa diakronikoak egin dituzten autoreek kontsideratu izan dute, atzizki menderagailuen oinarrian agertzen den -(e)n. hain segur, kasu marketarik heldu dela. Genitiboari dagokiola baieztatu dute Gavelek (1929) eta N'Diayek (1970), inesiboari, berriz, de Rijkek (1972) eta Jacobsenek (1977), morfo-fonologia kontuan harturik. Dakigun bezala atzizki hura da kausitzen erlatiboetan, zeharkako galderetan, perpaus subjonktiboetan, bai eta, beste zenbait atzizki harturik, beste forma askotan ere, agerian ( $(e) n$-ean, (e)n-etik,...).

Halere, menderagailu guziak ez dira atzizki, baititugu beste batzuk aurrizki moduan agertzen zaizkigunak. Hauek diakronikoki ez dira kasu paradigmari lotuak, baina enontziazioari, eta bereziki baieztapen markatzaileari (Lafon 1966). Hor ditugu. alde batetik, ha- baldintzetako protasietan euskalki guzietan baliatzen dena, eta bestetik, hait- egun ekialdeko euskalkietan bereziki baliatzen dena, baina 16 . mendean bizkaieraz ere agertzen dena bai(st)- forman.

Beste aurrizki bat sar daiteke sail horretan. usaian gramatikariek horrela egiten ez badute ere : inperatiboan agertzen den $b(e)$-aurrizkia, hain zuzen. Gutiz gehienetan, $b(e)$ - aurrizkia aurkezten da $d-$, z-, eta $l$ - denbora-moduzko aurrizkiak agertzen diren testuinguru morfologikoan, eta horregatik paradigma berean sartua izan da. Ez du iduri. haatik, egoki dela horren egitea, ezen aurrizki horren ezaugarri sintaktikoak kontuan hartzen badira, argi eta garbi ikusten da menderagailuen bereizgarriak dituela, eta ez denbora-moduzko markatzaileen(a)k. Bi argumentu eman daitezke honen erakusgarri :

- $b(e)$ - aurrizkiak ez ditu onartzen forma alokutiboak ;

- b(e)- aurrizkiak ez daiteke ager beste menderagailu batekin batean (aurrizkia edo atzizkia izan dadin). 
Bi ezaugarri horiek menderagailuek badituzte, ez, aitzitik, denbora-moduzko markatzaileek. Bestalde morfologikoki ere ez dirudi $b(e)$-, arras, $d-$, $z-$, eta $l$ - denboramoduzko aurrizkiekin pareka daitekeela. Alabaina, 18. eta 19. mendeko lapurterazko testuetan inperatiboko aurrizkia, hastean marka pertsonala duten adizkerekin ere aurkitzen da. Adibidez, nihork ez bezaitzate kondena dio Haranederrek (Kolos. 2, 16), adizkera horretan be-, $z$ - pertsona-aurrizkia duen zaitzate adizkerari lotua izanik. Dakigun bezala, hori guziz ezinezkoa denbora-moduzko aurrizkiekin, hauek aitzinean pertsona markarik ez duten adizkeretan baizik ez baitaitezke ager, salbuespenik gabe (*dazaituzte). Beraz, hirugarren argumentu hau ere eman daiteke, $b(e)$-aurriziki menperatzaileen artean sarzeko:

- b(e)- aurrizkia erabilia izan da zenbait euskalkitan pertsona-aurrizkiak zituzten adizkerekin ere.

Aurrizki menderatzaile bat inperatiboan agertzea ez da batere harrigarri. Lehenik, hizkuntza anitzetan defektiboak direlakotz inperatiboko formak, bereziki 2. pertsona subjektua ez den formetan (gaur egun euskalki gehienetan $b$-aurrizkia agertzeko betea izan behar den baldintzetarik bat ${ }^{2}$ ) ; bigarrenekorik euskara zaharrak gisa bereko lekukotasunak ematen dizkigulakotz.

Hain zuzen ere, Etxepareren olerkietan eta Leizarragaren itzulpenetan oinarrituz, lekukotasun horiek aurkeztuko ditugu hemen hiru aurrizki bereziki aztertuz : alha-, albait- eta ai-. Aipamen guti izan dute aurrizki horiek gure gramatiketan, salbu Lafonen tesian (1943:477-78,49 1-495), eta horregatik denen biltzera eta sailkatzera entseiatu gara.

Beraz, hiru aurrizkiak aldizka aurkeztuko ditugu, aldi bakoitz haien agertzea mugatzen duten baldintza morfosintaktikoak zehaztuz, bai eta haien balioa ere. Aipuak emanen ditugu, Leizarragarenen ondotik 1564 eko Genèveko testamentu berriaren frantsesezko testua ere eskainiz, bai eta Haranederren itzulpena ere (aditz forma bera itzulia denean bederen). Frantsesezko testu hori zergatik hartu dugun argi da : Leizarragak frantses testua izan zuen eredu bere itzulpena egiterakoan (Ruiz Arzallus 1991), eta 1564 eko argitalpen bat harturik, pentsa dezakegu Leizarragak erabili zuen bertsioa izan gabe ere, ez zatekeela biziki urrun ${ }^{3}$. Hala erakustera ematen ere dute hemengo itzulpenek. Haranederren erreferentzia aukeratu dugu, denboran hurbilena baitzen. Ordean, 170 urteko aldea bada bi itzulpenen artean, eta kontuan izanik jatorrizko bertsioak ere ez zirela berak, ez dezakegu konparantza horretarik ondorio handiegirik atera. Halere, konparantzak aurrizkien galera agerian emateko balio duela uste dugu.

\section{ALBA - aurrizkia}

Alba- aurrizkia hiru aldiz agerzen da Leizarragaren testuetan, eta sei aldiz Etxeparerenetan.

Adizkera trinkoekin (erran, egin, ${ }^{*} e d u n$ ) eta adizkera laguntzaileekin aurkitzen da. Azken kasu honetan, adibideen arabera behintzat, aditz lexikala aspektu markaduna bada, aspektu burutua izan behar du. Hots, ez dugu aurkitzen alba-aurrizkia, aditz lexikalak -tzen (edo are gutiago - $t u k o$ ) aspektu marka dakarren kasuetan ${ }^{4}$.

Alba- duten adizkera trinkoak, eta *edin eta *ezan laguntzaileak, hipotetikoan dira. salbu kasu batean, eta orduan partizipio perfektuarekin erabilia da (ik. (1)).

Subjektuak lehen $(4,9)$, bigarren $(5,7)$ edo hirugarren $(1,8)$ pertsonak izan daitezke. 
Alha aurrizkia $a l+b a$ morfemez osatua da, eta haren balioa elkarketa horri dagokio : alde batetik. ahala eta, beste aldetik, baldintzako suposizioa adierazten baitu, hala nola ahal ba- erabiltzen delarik. (3) adibidean ikus daiteke nola Leizarragaren egin alba dadi dioenean (Errom. 12,18), eguin ahal badadi ematen duen Haranederrek, bi formen baliokidetasuna agerian emanez. Adibide anitzetan, halako agiantza kutsu bat badute adibideek, supositiboarekin maiz gertatzen den bezala (cf. balinba).

Ikusirik aurrizkiak metatzen dituela haren barnean agertzen diren bi morfemen balioak, batek galde egin dezake ea egiazki aurrizki baten aitzinean gauden. Alabaina hegoaldeko testuetan guziz hedatua izan da al eta $b a$ - modu berean elkarri loturik eta aditzari erantsirik baliatzeko ohitura, bai testu zaharretan, bai eta, adibidez, Larramendiren gramatikan ere. Eta haien kasuan alba ez da aurriki gisa kontsideratzen, baina bi morfema desberdin gisa. Zergatik ez gauza bera egin hemen ? Arrazoi bakar batengatik : ez Leizarragak eta ez Exxeparek ez dute sekula silaba bakarreko eta aspiraziorik gabeko formarik erabiltzen ahal adierazteko, salbu alba-morfoarekin. Auziaren trinkatzeko bide segura izanen genuen, agertu izan balira ezezkako adibideak. Orduan, ezen, ikusiko zen $e z$ non agertuko zen : alba- aurrizkiaren aitzinean (ezalba-), ala ba- aurrizkiaren aitzinean (al ezha). Lehen kasuan, argi geldituko zen alba morfema konplexua izanagatik bat zela, hala nola gertatzen baita albait- morfemarekin (ik. §2) ; bigarren kasuan, alderantziz, frogaturik geratuko zen bi morfemak zirela, hegoaldeko tradizioan gertatzen den bezala. Zorigaitzez ezezkako adibiderik ez dugu aurkitu, eta, beraz, frogabide hori ezin baliatuzkoa dugu hemen.

Erran behar da, ordean, gure testuetan ahal morfema agertzen dela supositiboetan, eta beraz ez dezakegula pentsa ahal supositiboetan hartzen duen forma dela al. Hona Leizarragaren bi adibide :

Baina deus ahal badaguic (Mark. 9, 22)

Baldin hori sinhets ahal badeçac (Mark. 9,23)

Segurtamenik ez badugu ere, horrek alba- bestelako formatzat hartzera bultzatzen gaitu, hala nola egin baitzuen Lafonek (1943) ere.

\subsection{ALBA aurrizkia aditz jokabide perifrastikoan.}

- ALBA + *EDUN [+HIP].

ALBALUTE : egin albalute

(1) Eta eguna ethorri cenean, herria etzeçaten eçagut : baina portu itsas adar çuen bati ohart cequizquión, hartara, eguin albalute, vncia egotzi aiher ciraden. (Apos. eg. 27, 39)

1564ko Genèveko biblia: Et le iour venu ils ne cognurent point le pays: mais apperceurent un golphe avant riuage, auquels ils deliberovent ietter le nauire s'ils eussent pu.

Haraneder: eta gogo çuten untciari han joaracitceco, ahal baceçaten.

- ALBA + IZAN [+HIP].

ALBALIZ : izan albalitz

(2) Ceric cen bada çuen dohainontassuna ? ecen testificatzen drauçuet, baldin possible içan albaliz, çuen beguiac idoquiric eman cendrauzquedetela. (Galat. 4, 15) 
$1564 \mathrm{ko}$ Genèveko biblia: Quelle estoit donc vostre beatitude? car ie vous porte témoignage que s'il eust este possible, vous eussiez arrache vos yeux. \& les m'eussiez donnez.

Haraneder: Non da bada orduco çuen dohatsutasuna? Ecen lekhukotassun hau errenda dieçaqueçuet, possible içan balitz çuen beguiac iraciric emanen cinarozquitedala.

- ALBA + *EDIN [+ ORAIN].

ALBADADl : egin albadadi

(3) Eguin albadadi, çuetan den becembatean, guiçon guciequin baquea duçuen. (Errom. 12.18)

$1564 \mathrm{ko}$ Genèveko biblia: Si se peut faire, en tant qu'en vous est, avez paix avec tous hommes.

Haraneder: Eguin ahal badadi eta f̧uen baitan dagoen guçiaz, duçuen baquea guiçon guciequin.

- ALBA + *EDIN [+HIP].

ALBANENGIDIO : parti albanengidio

(4) Parti albanenguidio harc ezluque pareric/Alavana nic ezticit hayn hon deriçadanic. (Amorosen partizia)

- ALBA + *EZAN [+HIP].

ALBAHEZA : eskusa albaheza

(5) Vercen gaztigari inçan oray adi gaztiga/Pena honez orhit eta hangoa ezac cogita/Hebengoaz vercecoa albaheça escusa/Vnsa enplegatu duquec heben eure denbora. (Mossen Bernat echaparere cantuya)

ALBANEZA : itzul albaneza

(6) Elas yzul albaneça yragan den denbora/Segur oray enyqueci dudan gogoan veharra. (Amorosen partizia)

1.2. ALBA aurrizkia aditz jokabide trinkoan. :

- ALBA + EGIN [+ORAIN].

ALBADAGIK

(7) Albadaguic ioan adi eliçara goycian/leyncoari han gomenda bere eche saynduyan. (Doctrina Christiana, Goycian)

- ALBA + *EDUN [+ORAIN]. ALBADITU

(8) Hirur gauça albaditu ehorc ere eguiaz/Nola ere hil vaytadi doha saluamenduyan. (Doctrina Christiana, Harmac eryoaren contra)

- ALBA + ERRAN [+HIP]. ALBANERRA

(9) Albanerra eguya nyc dut pena handia/Secretuqui minça guiten bioc othoy maytia. (Amorez errequericia.) 


\section{Albait- aurrizkia.}

Albait-aurrizkia alba-baino gehiago agertzen da 16 . mendeko testuetan. Leizarragaren testuetan 27 adibide bildu ditut. eta 8 Etxeparerenetan.

Albait-aurrizkia morfologikoki aitzinekoaren irudikoa da, ezen elkarretaratzen ditu al morfema eta aditz aurrizki bat, kasu honetan ez ba-supositiboa, baina bait-. Haatik, guziz desberdina da semantikoki : al morfemak ez du ahalezkoa deusetan adierazten, eta aurrizkia agintera irudiko perpausetan agertzen da, Lafonek, horrengatik, prescriptif deitu baitzuen. Bestalde, albait- ez da edozein alditako adizkerekin baliatzen, baina hipotetikoan direnekin bakarrik.

Albait- bi formatan agertzen zaigu : diptongoa hetsiz darabil kasik beti Leizarragak (albeit-), hetsi gabe Etxeparek (albait-) $)^{3}$. Aldaketa hori guziz ezaguna da bait morfemaren kasuan.

Albait-morfema konposatua bada, aurrizki bakarra da. Hori erakustera ematen digute ezezkoan diren hamar bat adibideek (salbuespenik gabe). Horrelakoetan $e z$ morfema albait- aurrizkiari juntatzen zaio (ez bait- morfemari) : eta campoetan diradenac ezalbeilitez hartan sar (Luk. 21, 21).

Lafonek oharrarazi zuen albait-noiz baliatzen zen : pour exprimer un ordre dont l'exécution n'est subordonnée à aucune condition et doit avoir lieu immédiatement.. Quand le verbe est au prescriptif. l'éxécution du procies voulu, ordonné par le sujet parlant n'est pas rapportée au présent immédiat : tantôt, subordonnée à l'accomplissement d'un autre procès qui n'est pas d'ores et déjà réalisé, elle concerne de ce fait l'avenir, tantôt elle dépasse et déborde le moment présent parce que le verbe exprime une prescription permanente, qui peut être catégorique ou subordonnée ellemême à une condition. (1943:491)

Lafonek errana adibide gehienetan egiaztatzen da. Adibidez :

- - Perpauseko subjektua (edo beste osagai argumental bat), solas ingurumenaz beste egoera bati dagokion erlatibo libro batek gorpuzten du (cf. (35), (36), (37)), edo erlatibo korrelatu batek (cf. (20), (24), (34), (39)) :

Orduan Iudean diradenéc ihes albeileguite mendietarát (Luk. 21, 21); (37).

Eta norc-ere nahi vkanen baihau bortchatu lecoa baten eguitera, albeitindoa harequin biga. (Mat. 5, 41); (34).

- Agindua denbora markatzaile bati lotua da eta honek haustura markatzen du solas ingurumenaren aldetik. Denbora markatzailea izan daiteke : denborazko perpaus menderatua (cf. (17), (18), (22), (23), (25), (42) (43)), edo denborazko izen sintagma hala nola bertze aldian (cf. (19)), egun hartan (cf. (21)), orduan (cf. (43)) :

Igor deçadanean Artemas hiregana edo Tichique, diligenta albeitendi enegana ethortera Nicapolisera (Tito 3, 12); (17).

Aleguera albeitzinteizte egun hartan, eta bozcarioziauz. (Luk. 6, 23) ; (21).

- Agindua baldintza baten konplitzeari darraio (cf. (28), (41)) :

Eta orduan baldin nehorc badarraçue. Huná hemen Christ, edo. Hará han : ezalbeitzineçate sinhets. (Mark. 13,21): (28).

Hala ere. ez dirudi Lafonek azpimarratu puntu hori ezin besteko baldintza dela albait- enplegatzeko. Alabaina Etxepareren testuetan badira adibide batzuk aipatu 
azterbideari bizkar ematen diotenak. Horrela Potaren galdacia olerkian dialogoak zuzenean ekarriak dira, eta hala-hala andereak pot galdez dagokionari erranak (ik. (30), (33)) :

Horrelaco hiz gaixtoric ning eztarradala/Vercer erran albaytica enuc vste duvana. (Potaren galdacia.) ; (33).

Nekez holako adibide baten kasuan erran daiteke agindua solas egoeratik kanpo kokatzen dela.

Dena dela ongi azpimarratu behar da albait-ekilako inperatiboak eta bestelakoak ez direla banaketa konplementarioan. Koordinazioa gertatzen denean, ikus dezakegu batzuetan albait-ekilako formak koordinatzen direla (ik. adibidez (15), (16), (22),...), eta beste batzuetan, berriz, inperatibo arruntak (ik. (10), (26), (33),...). Hona bi koordinazio moten adibide bedera :

Eta gauça hauc eguiten has ditecenean, chuchent albeitzinteizte, eta goiti albeitzinçate fuen buruäc, ecen hurbiltzen da fuen redemptionea. (Luk. $21,28) ;(22)$.

Eta hatzamanic eramanen f̧aituztenean etzaretela aitzinetic ansiatan cer erranen duçuen, eta ezalbeitzineçate medita (Mark. 13, 11) ; (26)).

Alhait- aspektu markarik ez dakarten adizkerekin agertzen da : edo *edin eta *ezan aditz laguntzaileei loturik aditz nagusiak aspektu markarik ez izanez, edo adizkera trinkoekin. Bi baldintza badira albait- agertzeko:

- adizkera hipotetikoan izan behar da ;

- subjektua 2. edo 3. pertsona izan behar da.

Ohar bedi subjektua 2. pertsona denean ere hipotetikoa agertzen dela albait-aurrizkiarekin. eta ez orainaldiko adizkera bat. Beraz kontraste garbia dugu puntu horretan inperatibo arruntekin : so albaitegi (= so egin ezak), utzi albainenzak (= utzi nazak), albeitindoa $(=(h) o a)$, etab.

\subsection{Albait- aurrizkia aditz jokabide perifrastikoan.}

\subsubsection{Albait- aurrizkia *edin aditz laguntzailearekin.}

- ALBAIT + LEDI : ALBAILEDI

- albailedi segura

(10) Vercen gayçaz cençacia çuhurcia handi da/Yzterbegui duyen oro nitan vedi gaztiga/Abantallan dabilela albayledi segura. (Mossen Bemat echaparere cantuya)

- ezalbeiledi jauts

(11) Eta etche gainean datena, ezalbeiledi iauts deusen bere etchetic eramaitera. (Mat. 24, 17)

1564 ko Genèveko bihlia: Et celù qui sera sur la maison ne descende point pour emporter aucune chose de sa maison.

Haraneder: etche gaiñean ditequena ez bedi jauts etchetic deussen eramaterat

(12) Eta etche gainean datena, ezalbeiledi iauts etcherát, eta ezalbeiledi sar deusen bere etchetic eramaitera. (Mark. 13, 15) 
$1564 \mathrm{kn}$ Genèveko biblia: Et celuy qui sera sur la maison, ne descende point en la maison, \& n'y entre point pour emporter aucune chose de la maison. Haraneder: etche gaiñean dena ez bedi jauts etcherat, ez barnerat sar, cerbait eramateco guraz.

- ezalbeiledi itzul

(13) Eta landán datena, ezalbeiledi guibelerat itzul bere abillamenduén hartzera. (Mat. 24, 18)

$1564 k 0$ Genèveko biblia : Et celuy qui est au champ, ne retourne point en arriere pour emporter les habillemens.

Haraneder: eta larrean içanen dena ez bedi bihur bere arrobaren hartcerat.

(14) Eta landán datena ezalbeiledi guibelerat itzul, bere abillamenduaren hartzera. (Mark. 13. 16)

1564ko Genèveko biblia: Et celuv qui sera au champ. qu'il ne retourne point en arriere pour emporter son vestement.

Haraneder: eta landan ditequena ez bedi bihur guibelat bere tresnen hartcerat.

(15) Egun hartan etche gainean datena, eta bere ostillamendua etchean badu, ezalbeiledi iauts haren eramaitera : eta landán dena, halaber ezalbeiledi itzul guibelecoetara. (Luk. 17, 31)

1564ko Genèveko biblia: En ce jour-la qui sera sur la maison, \& aura son mesnage en la maison, qu'il ne descende point pour l'emporter : \& qui sera és champs, semblablement ne retourne point à ce qui lui est laissé derriere.

Haraneder: Ordu hartan etche gaiñean ditequena eta bere mubleac etchean dituena, ez bedi jauts hequien eramaterat; eta landan ditequena ez bedi bihur guibelat.

- ALBAI (T) + LITEZ : ALBAILITEZ

- retira albeilitez, ezalbeilitez sar

(16) : Orduan ludean diradenéc ihes albeileguite mendietarát : eta haren arecoac retira albeilitez : eta campoetan diradenac ezalbeilitez hartan sar. (Luk. 21. 21)

1564ko Genèveko hiblia: Alors que ceux qui sont en Iudée. qu'ils s'enfuvent aux montagnes: \& ceux qui sont au milieu d'icelle, qu'ils se retirent: \& que ceux qui sont és champs n'entrent point dedans icelle.

Haraneder: Orduan Judean direnec ihes eguin beçate mendietarat eta herriaren erdian direnac biohaz urrun eta ingurutaco herritan direnac ez daitecela hartan sar.

- ALBAIT + (H) ENDI : ALBEITENDI

- diligenta albeitendi

(17) : Igor deçadanean Artemas hiregana edo Tychique, diligenta albeitendi enegana ethortera Nicapolisera : ecen han neguären iragaitera deliberatu diát. (Tite 3. 12)

$1564 \mathrm{ko}$ Genèveko biblia: Quand j'enuoroveray vers toy Artemas ou Tichique, diligente-toy de venir vers moñ à Nicopolis : car i'an deliberé de 
faire là mon hiver.

Haraneder: Igorri darotçuquedanean Artemas eta Tichique, khecha çaite eneganat ethorcerat Nicopoliserat, ecen han iragan gogo dut negua.

- orhoit albeitendi

(18) Eta erran cieçón Iesusi, Orhoit albeitendi niçaz, launa, ethor adinean eure resumara. (Luk. 23, 42)

1564ko Genèveko biblia: Et disais à iesus, Seigneur aye memoire de moy quand tu viendras en ton regne.

Haraneder: Guero Jesusi erraten cioten : "Jauna, orhoit çaite nitaz çure erresuman sarthu çaitezquenean.

- ALBAIT + ZINTE : ALBAITZINDE

- mintza albaizinde

(19) : Etay lelo rybay lelo pota franco vercia vego/Andria minça albaycinde verce aldian emiago. (Etchepare, Potaren galdacia)

- ALBAIT + ZINTEZTE : ALBAITZINDEZTE, ALBEITZINTEIZTE

- ilki albaitzindezte ${ }^{6}$

(20) Eta cein-ere etchetan sarthuren baitzarete, han çaudete, eta handic ilki albaitzindezte (Luk. 9, 4)

$1564 \mathrm{ko}$ Genèveko biblia: Et en quelconque maison que vous entriez, demeurez$x, \&$ vous en allez de là.

Haraneder: Cein ere etchetan sarthuco baitçarete, han egon çaitezte eta ez çaiteztela ilkhi handic

- alegera albeitzinteizte

(21) A leguera albeitzinteizte egun hartan, eta bozcarioz iauz. (Luk. 6, 23)

1564ko Genèveko biblia: Esiouissez-vous en ce jour-la. et sautez de iove. Haraneder: Orduan çaitezte aleguera eta bozcaria.

- xuxent albeitzinteizte

(22) Eta gauça hauc eguiten has ditecenean, chuchent albeitzinteizte, eta goiti albeitzinçate çuen buruäc. (Luk. 21, 28)

$1564 k n$ Genèveko biblia: Or quand ces choses commenceront à ce faire : dressez-vous en haut, \& leuez vos testes.

- ezalbeitzinteizte izit

(23) Eta ençun ditzaçuenean guerlâc eta seditioneac ezalbeitzinteizte icit. (Luk. 21, 9)

1564 ko Genèveko biblia: Et quand vous orrez des guerres \& seditions, ne vous espouantez point.

Haraneder: Adituco duçuenean, bada, mintçatcen guerlaz eta alboramenduz. ez çaiteztela espanti. 


\subsubsection{Albait- aurrizkia *ezan aditz laguntzailearekin}

- ALBAIT + ZINEZATE : ALBEITZINEZATE

- iharros albeitzinezate

(24) Eta norc-ere recebituren ezpaitzaituzte, eta ez çuen hitzey behaturen, etche edo hiri hartaric ilkitean iharros albeitzineçate çuen oinetaco errhautsa. (Mat. 10, 14)

1564ko Genèveko biblia: Et quiconque ne vous receura \& n'escoutera vos paroles, partans de la maison ou de la ville, secouez la poudre de vos pieds.

Haraneder: Eta norbaitec errecibitu nahi ez çaituztenean eta çuen hitçac aditu nahi ez dituenean, etchetic edo hiritic ilkhitçearequien batean, iharros çaçue f̧uen oiñetaco herrautsa.

- ezagut albeitzinezate

(25) Halaber çuec-ere ikussiren duqueçuenean gauça hauc eguiten diradela, eçagut albeitzineçate ecen hurbil dela Iaincoaren resumá. (Luk. 21, 31)

$1564 k o$ Genèveko biblia: Pareillement aussi quand vous verrez ces choses estre faites, sachez que le regne de Dieu est pres.

Haraneder: Hala fuec ere icusten dituzquetçuenean gauf̧a horiec guertatcen, jaquiçu hurbil ditequela Jaincoaren erresuma.

- ezalbeitzinezate medita

(26) Eta hatzamanic eramanen çaituztenean etzaretela aitzinetic ansiatan cer erranen duçuen, eta ezalbeitzineçate medita (Mark. 13, 11)

$1564 \mathrm{ko}$ Genèveko hihlia: Quand donc ils vous meneront pour vous liurer. ne sovez deuant en souci de ce que vous direz. et \& n'y medites point.

- ezalbeitzinezate sinhets

(27) Orduan baldin nehorc badarraçue, Huná hemen Christ, edo han : ezalbeitzineçate sinhets. (Mat. 24, 23)

$1564 \mathrm{ko}$ Geneveko hiblia: Lors si quelcun vous dit. Voici le Christ, ici ou là, ne le croyez point.

Haraneder: Baldin orduan cembaitec erraten badarotcue : Huna Christo hemen da et (a) horra hor da, ez deçaçuela sinhetx.

(28) Eta orduan baldin nehorc badarraçue, Huná hemen Christ, edo, Hará han : ezalbeitzineçate sinhets. (Mark. 13,21)

1564ko Genèveko hiblia: Alors donc si aucun vous dit, Voici le Christ ici, ou le vola, ne le croyez point.

Haraneder: Orduan baldin nihorc erraten badarotçue: Huna non den Christo, edo, Horra non den. ez deçaçuela sinhetx.

- ALBAIT + ZIN(I)TZATE : ALBEITZINTZATE

- goiti albeitzintzate

(29) Eta gauça hauc eguiten has ditecenean, chuchent albeitzinteizte, eta goiti albeitzinçate çuen buruäc, ecen hurbiltzen da çuen redemptionea. (Luk. 21, 28)

$1564 k o$ Genèveko biblia: Or quand ces choses commenceront à ce faire : dressez-vous en haut, \& leuez vos testes: car vostre deliurance approche. 
Haraneder: Gauça horiec hasten direnean bada heltcen, idec fatciçue beguiac eta altcha buruac, cergatic hurbill baiditeque çuen errestecamendua

- ALBAIT + NENZAK : ALBAINENZAK

- utzi albainenzak

(30) Hire potac bacyaquyat berce gauça nahi dic/Anderia azti cira nihaurc erran gaberic/ Bada vci albaynençac ny holacoz yxilic/ Horreyn gayz ciraden guero eguinen dut verceric. (Potaren galdacia : 4)

- ALBAIT + (H)EZA : ALBAITEZA

- albaiteza konserba

(31) Honequila albayteça bethiere conuersa/Gaixtoequi ecin ayte gayzqui beci prouecha/Bercer eguin eztaçala nahi eçuqueyena. (Doctrina Christiana)

- egin albaiteza

(32) Eliçara içanian so eguic bateyarrira/Pensa eçac han duyala recebitu fedia/leyncoaren gracia eta saluaçeco vidia/Hari eguin albaiteça lehen eçagucia. (Doctrina Christiana, Batheyarria)

- ALBAIT + (H) ITZA : ALBAITITZA

- erran albaititza

(33) Horrelaco hiz gaixtoric niry eztarradala/Vercer erran albaytiça enuc vste duyana. (Potaren galdacia.)

\subsection{Albait- aurrizkia aditz jokabide trinkoan}

\subsubsection{Joan aditza}

- ALBAIT + (H)INDOA : ALBEITINDOA

(34) Eta norc-ere nahi vkanen baihau bortchatu lecoa baten eguitera, albeitindoa harequin biga. (Mat. 5, 41)

$1564 k o$ Genèveko biblia: Et quiconque te voudra contraindre d'aller une lieuë, vas-en deux auec luy.

Haraneder: Eta nihoc bortchatu nahi baitçaitu harequien goaterat milla pausu. cohazco bence bi mila pausu ere.

2.2.2. Egin aditza

- ALBAI(T) + LEGITE : ALBEILEGITE

- ihes albeilegite

(35) Orduan Iudean diratenéc, ihes albeileguite mendietarát. (Mat. 24, 16)

$564 k o$ Genèveko biblia: $A$ donc que ceux qui seront en Iudée, s'enfuyent aux montagnes.

Haraneder: Orduan Judean direnec ihes eguin beçate mendietarat. 
(36) Dacussaçuenean bada desolationearen abominationea, Daniel Prophetáz erran içan dena, behar eztén lekuan dagoela (iracurtzen duenac adi beça) orduan Iudean diratenéc. ihes albeileguite mendietarát. (Mark. 13, 14)

$1564 k o$ Genèveko biblia: Or quand vous verrez l'abomination de la desolation (qui est dite par Daniel le Prophete) estre où elle ne doit (qui lit l'entende) alors que ceux qui seront en ludee. fuyent aux montagnes.

Haraneder:... orduan Judean içanen direnec eguin beçate ihes mendietarat.

(37) Orduan Iudean diradenéc ihes albeileguite mendietarát (Luk. 21, 21)

1564ko Gerèveko biblia: Alors que ceux qui seront en Iudee, qu'ils s'enfuyent aux montagnes.

Haraneder:... orduan Judean direnec ihes eguin begate mendietarat.

- ALBAIT + (H)EGI : ALBAITEGI

- so albaitegi

(38) Vertan guero so albaitegui non den gorpuz sainduya. (Doctrina Christiana. Gorpuz saynduya)

\subsubsection{Erran aditza}

- ALBAIT + ZINARRATE : ALBEITZINARRATE

(39) ALBEITZINARRATE : baina cer-ere emanen baitzaiçue ordu hartan, hura albeitzinarrate : ecen etzarete çuec minço çaretenac, baina Spiritu saindua. (Mark. 13, 11)

$1564 \mathrm{ko}$ Genèveko bihlia: mais tout ce qui vous sera donné en cet instantla, dites cela : car ce n'estes-vous qui parlez, mais le saint Esprit.

Haraneder: bainan cer ere ordu hartan berean emanen baitçaitçue, eta hura erraçue

(40) Ihardets ciecén, Othoitzeric eguinen duçuenean, albeitzinarrate hunela : Gure Aita... (Catech. Orationeaz).

\subsubsection{Jakin aditza}

- ALBAIT + LEKI : EZALBEILEKI, ALBALIAKI

- ezalbeileki

(41) Baina hic elemosyna eguiten duanean, ezalbeilequi hire ezquerrac, cer eguiten duen hire escuinac. (Mat. 6,3)

1564 ko Genèveko biblia: Mais quand tu fais aumosne, ta senestre ne sache ce que fait ta dextre.

Haraneder: Cuc, bada, eguiñen duçunean amoiña, ez defan jaquin (sic) çure escu ezquerrac cer eguiten duquen çure escuiñeco escuac.

- albailiaki

(42) Eta hoyec eguiazqui ehorc hala ezpaditu/Albayliaqui duda gabe ecin dateyela salbu. (Doctrina Christiana) 
- ALBAIT + ZINEKITE : ALBEITZINEKITE

(43) : Hala çuec-ere dacusquiçuenean gauça hauc guciac, albeitzinequite écen borthan hurbil datela. (Mat. 24, 33)

$1564 k o$ Genèveko hiblia: Vous aussi pareillement, quand vous verrez toute ces choses, sachez qu'il est prochain à la porte.

Haraneder: hala fuec ere horiec guciac icusten dituzquetçuenean, jaquiçue Guiçonaren Semea hurbill eta athean ere ditequela.

(44) Eta ikus deçaçuenean Ierusaleme gendarmeriaz inguratua, orduan albeitzinequite ecen hurbil datela haren destructionea. (Luk. 21, 20)

1564 ko Genèveko biblia: Et quand vous verrez Ierusalem estre enuironnee d'armees, sachez adonc que sa desolation est prochaine.

Haraneder: Icussico duçuenean. bada, Jerusalem armada batec inguratcen duela. jaquin çaçue hurbill ditequela haren desolacionea.

\section{3. $A i$ - aurrizkia}

$A i$ - aurrizkia gaur egun guziz zaharkitutzat edo hiltzat eman behar badugu ere, lehen aipatu bi aurizkiek baino luzezago iraun du ekialdeko euskalkietan. Hala iduri du behinik behin.

Joan den mendeko zuberotar gramatikariek ematen digute haren berri : bai Chahok (1836: 158), bai Inchauspek (1858: 105-113), bai Gèzek (1873:167-8, 191-2), erran gabe hila zela edo desagertzen hasia zela. Bonapartek ere bere Le verbe basque en tableaux $(1869,183$. o. aldien bildumako taulari mugatuz gero) ematen ditu forma hauek, temps n'appartenant qu'au souletin direla gaineratuz, gero.

Orain testuei berei behatzen hasten garenean bizitasun horren hatzik ez dugu kausitzen batere. Ez ditugu eiki testu guziak miatu, baina anitz bai, eta egiaren aitortzeko, ondorioak ez dira gramatikek erranak ikusiz iguriki zitezkeenak.

16. mendean, Etxeparek ez zuen erabili, bai, ordean Leizarragak, baina guti (lau adibide). 17. mendean, Axularrek bi aldiz erabili zuen eta Oihenartek ere berdin. S. Pouvreauk, berriz, anitz, eta kasu batzuetan berak bakarrik eskaintzen dituen forma batzuekin. Antza denaz, ez Tartasek eta ez Belapeyrek ez zuten baliatu, idazle horien argitaratzaileek ez baitigute seinalatzen ( P. Altuna, P. Agirre). Gero, 17. mendearen ondotik Mirande arte (erran gabe doa Mirandek liburuetan ikasia zukeela) ez dugu inon kausitzen, salbu aipatu gramatika lanetan.

Chahoren lekukotasuna garrantzi handikoa da. Berak zubererazko paradigmak eskaini zituen bere gramatikan, ai- aurrizkiarekilako formak izan (NOR bakarrean) eta * edun (NOR-NORK) aditz paradigmen arabera emanez (eta adibiderik gabe). Nola aurreko gramatika lanetan ez baita batere aipatzen ai-, eta nola zubererazko testuetan ez baita agertzen (Oihenarten bi adibide haiek izan ezik, ik. 6. oh.), pentsa dezakegu ahozko mintzairatik ezagutzen zuela Chahok. Bonaparteren erranak ere hipotesi honen berme dira, nahiz bitxi baino bitxiago den ikustea zein guti baliatua izan duten beren testuetan zuberotarrek hango gramatikariek aipatu morfema hori.

16. mendean ai- agertzen da albait bezala adizkera hipotetikoetan, edo *edin eta * ezan aditz laguntzaileekin, edo aditz trinkoekin. Ez zatekeen hori ezin besteko baldintza, Oihenarten adibideek erakustera ematen duten bezala. Alabaina honek ez ailiz jaio forma erabili zuen, izan aditz laguntzailearekin eta aditz nagusia aspektu burutuan izanik (ik. 6. oharra). 
Haren balioa agiantzari dagokio eta botibo moduan definitua da. Duvoisinek, adibidez, edo Haranederrek, oxala edo balinba erabiliko dute zenbaitetan ingurumen beretan. Leizarragaren lau adibideen frantsesezko bertsioan, berriz, à la mienne volonté espresioa kausitzen dugu, iraganaldiko edo perfektuaz haragoko subjonktiboko forma batekin : aihinz 'à la mienne volonté que tu fusses'(47).

Ohargarria da, halaber, aditz jokatua aitzinean emana dela, jokabide perifrastikoan aditz nagusia baino lehenago (ailitez trenca, ainençaçue supporta, aitzineçate regna) eta kopulari dagokionean, predikatua baino lehen (aihinz hotz edo eraquin).

Gure 16. mendeko adibideetan holakorik agertzen ez denarren, subjektua edozein pertsona izan daiteke, lehena ere bai. Horrela diote 19. mendeko gramatika guziek. Egia errateko adibide guti da testuetan holakoa denik. Kausitu direnak Pouvreaurenak dira, hala nola hau :

Hala ere aineza oren erditsu hura bedere behar bezala iragan! (Iesusen imitazionea, 246. o.).

Bururatzeko derragun, kasu batean izan ezik, ai- dela beti aurrizkia. (48) adibidean ait- agertzen da (aitzineçate). Haatik, hapax denaz gero, hutsa izan daiteke edo baitekilako analogiaz agertu forma bat.

\subsection{AI + IZAN (trinkoa)}

\section{- AIHINZ}

(45) Baceaquizquiat hire obrác, ecen ezaicela ez hotz ez eraquin : aihinz hotz edo eraquin. (Apok. 3, 15)

1564ko Genèvęo hiblia: A la mienne volonté que tu fusses froid ou bouillant.

Haranederm : Badaquizquit fure eguitateac, ez faretela ez hotz, ez bero. Ochala hotza baf̧iñe edo beroa.

\section{2. $\mathrm{AI}+* \mathbf{E D I N}$}

- AILITEZ : ailitez trenka

(46) Ailitez trenca çuec trublatzen çaituztenac. (Galat. 5, 12)

1564ko Genèveko biblia: A la mienne volonté que ceux qui troublent vostre repos, fussent retranchez.

Haraneder: Ochala ebakiak ere balire fuec alraratcen çaituztenac.

\section{3. $\mathrm{AI}+{ }^{*}$ EZAN}

- AINENZAZUE : ainenzazue suporta

(47) A inençaçue supporta appurbat neure erhogoán, baina aitzitic supporta neçaçue. (2 Korin. 11, 1)

$1564 k o$ Genèveko hiblia: A la mienne volonté que vous me supportissiez un petit en ma folie: \& certes vous me supportez.

Haraneder: Nahi nuque paira bacineçate aphur bat ene erguelqueria!

Duvoisin: Ochala aphur bat jasan bazinezate ene sendokeria! 
- AIZINEZATE : aitzinezate regna

(48) Ia asse çarete, ia abrastu çarete, gu gabe reguetu çarete : eta aitzineçate regna, guc-ere çuequin batean regna deçagunçát. (1. Korin. 4, 8).

1564ko Genèveko biblia: \& à la mienne volonté que vous ne regnissiez, afin que nous aussi régnions avec vous.

Haraneder: Ja asseac çarete, ja aberastuac çarete; gu gabe erreguñatcen duçue eta ochala erreguiñatcen bacindute guc ere cuequien batean erreguiña deçaguntçat.

B. OYHARÇABAL

UPRESA du CNRS 


\section{Bibliografia}

Itzultzaile edo itzultzaile izenik gabe, 1564. Nouueau Testament (reueu \& corrigé sur le Grec par les Ministres de Genève), chez Jean Crespin.

Abbadie, A. de - 1836. 'Prolégomènes', in A. Th. d'Abbadie \& J. A. Chaho, Etudes grammaticales sur la langue euskarienne, 1-50. o., Paris.

Axular, P ; 1643. Gero, L. Villasantek prestatu argitalpena, 1976, Jakin, Oñati.

Azkue. R. M. de -. 1923-25. Morfologia Vasca, 2. argitalpena, 1969, 2 liburuki, La Gran Enciclopedia Vasca, Bilbao.

Bonaparte, L.-L. 1869. Le verbe basque en tableaux, Euskaltzaindiaren argitalpena in Opera Omnia Vasconice, 1, 175-442. o., 1991, Euskaltzaindia, Bilbo.

Calvin, J. Catéchisme, 1553ko argitalpenaren edizio berria : Catéchisme de l'Eglise de Genève, 1853, Imprimerie Jules-Guillaune Fick, Genève.

Chaho, A. 1836. 'Grammaire euskarienne', in A. Th. d'Abbadie \& J. A. Chaho, Etudes grammaticales sur la langue euskarienne, 51-184. o., Paris.

Etxepare, B. 1545. Linguae Vasconum Primitiae, P. Altunaren edizio kritikoa, 1980, Lekukoak, 2, Euskaltzaindia \& Ed. Mensajero, Bilbo.

Gavel, H. 1929. Grammaire basque, 1. liburukia : Phonétique. Parties du discours autres que le verbe, Imprimerie du Courrier, Baiona.

Gèze, L. 1873. Eléments de grammaire de basque, fac-similezko argitalpena, 1979, Euskal klasikoak, 27, Hordago-Lur, Donostia.

Haraneder, J. de -. 1990 [1740]. Jesu Christoren evanjelio saindua, P. Altunaren edizio kritikoa, Lekukoak, 17, Euskaltzaindia, Bilbao.

Humboldt, W. von - 1817. Berichtigungen und Zusätze zum ersten Abschnitte des zweyten Bandes des Mithridates über die Cantabrische oder Baskische Sprache, J. Garateren gaztelaniazko itzulpena : Correciones y Adiciones al Mithridates de Adelung sobre la Lengua Cantabrica o Vasca, RIEV, 24-3, 1933, Donostia.

Inchauspe, Abbé. 1858. Le verbe basque, Baiona-Paris.

Jacobsen, W. H. 1977. 'The Basque Locative Suffix', in W.A. Douglass, R.W. Etulain \& W.H. Jacobsen (argit.) : Anglo-American Contributions to basque Studies : Essays in Honor of Jon Bilbao, 163-175. o., Desen Reseach Institute, Reno, Nevada.

Leizarraga, J. 1571. Iesus Christ gure Iaunaren testamentu berria ; Othoitza ecclesiasticoen forma ; Catechismea ; Kalendrera ; $A B C$ edo christinoen instructionea, T. Linschmann \& H. Schuchardtek prestatu edizioaren fac-similezko argitalpena, 1990, Euskaltzaindia, Bilbo.

Lafitte. P. 1944-62. Grammaire basque (dialecte navarro-labourdin, Bigarren argitalpena. zuzendua eta aberastua, 1962, Amis du Musée Basque eta Ikas, Baiona.

Lafon, R. 1943. Les formes simples du verbe basque dans les principaux textes du l6ème siècle, Delmas, Bordeaux.

Lafon, R. 1966. 'La particule bait- en basque : ses emplois morphologiques et syntaxiques. BSL, LXI, 217-240. o. Paris.

Michelena. L ; 1978. 'Miscelanea filologica vasca, $1 F L V, 10,205-288$. o. 
N'Diaye, G. 1970. Structure du dialecte basque de Mava, Mouton, La Haye-Paris.

Oihenart, A. d'-.1657. Atsotizac edo Refravac, $O .{ }^{\mathrm{m}}$ gastaroa nevrthizetan, J.-B. Orpustanek prestatu hiru hizkuntzatako argitalpena : Proverbes et poésies basques, 1992, Izpegi, Baigorri.

Pouvreau, S. 1979 [ $\infty 1660]$. Iesusen imitationea, Euskal klasikoak, 25, Hordago-Lur, Donostia.

Rijk, R. P. de -. 1972. Studies in basque Syntax : relative clauses, Ph. D., MIT.

Ruiz Arzallus, I. 1991. 'El modelo griego de Leizarraga : una quimera filologica?', in J.A. Lakarra \& I. Ruiz Arzallus (argit.) : Memorice Mitxelena Magistri Sacrum, Pars prior, ASJUren gehigarriak, XIV, 107-115. o., Gipuzkoako Fon Aldundia, Donostia.

Schuchardt, H. 1923. Primitice Linguce Vasconum. Einfuirung ins Baskische, gaztelaniazko itzulpena: Tesis y estudios salmantinos III, 1947, Salamanca.

Van Eys, W. J. 1900-01. Bibliographie des Bibles et des Nouveaux Testaments en langue française des $15^{\circ}$ et $16^{\prime}$ siècles, Genève. 


\section{Résumé \\ A propos de trois anciens préfixes verbaux dans les textes du 16 16 $^{\circ}$ siècle}

En basque le marquage de la subordination des phrases conjuguées se réalise au moyen d'affixes se joignant aux formes personnelles des verbes. Ces affixes sont soit des suffixes, diachroniquement issus de marqueurs casuels, soit des préfixes, lesquels sont reliés à la particule d'affirmation positive bai 'oui'. Dans ce second cas, il s'agit de $b a$ - 'si' qui marque les protases de conditionnel, et bait- qui est un complémenteur utilisé dans diverses constructions, et qui peut prendre parfois une valeur causale. Il est proposé dans l'article, d'y joindre également $b(e)$, qui est utilisé à l'impératif lorsque le sujet est de $3^{e}$ personne, bien que ce morphème soit d'ordinaire analysé comme appartenant au paradigme des prefixes modaux-temporaux $(d-, z-, l-)$.

La vieille langue a laissé le témoignage dans les dialectes labourdins et bas-navarrais d'autres préfixes issus du marquage énonciatif ou modal. Ils sont associés à l'expression de la condition (alba-), de la prescription (albait-), ou du souhait (ai-). Ces préfixes, parmi lesquels les deux premiers surtout ont pris rapidement une allure archä̈ue, sont inconnus dans la langue moderne, et ont toujours eu, avant même de disparaitre, une position plus ou moins marginale.

Seul Lafon dans sa thèse sur le verbe au $16^{\circ}$ siècle avait prêté attention jusqu'ici à ces morphèmes. Aussi l'article reprend-il les données de manière exhaustive en se fondant sur les textes du $16^{\circ}$ siècle, et plus précisément de ceux de Liçarrague et Etchepare, car lesdits préfixes n'apparaissent dans aucun autre texte du $16^{\circ}$ siècle.

Chacun d'eux est successivement examiné, en indiquant les conditions morphosyntaxiques régissant son emploi, et sa valeur. Tous les exemples rencontrés sont cités suivant le classement des formes verbales préfixées. Pour chacune des formes employées par Liçarrague, et extraites de la traduction du Nouveau Testament, nous produisons la version française proposée par le catéchisme de Genève publié en 1564, car il restitue de fort près le principal texte source qu'a dû utiliser Liçarrague (la source précise n'est pas connue). Chaque fois que la forme préfixée est également traduite dans la version de Haraneder, nous joignons à titre de comparaison cette version. Bien que postérieure d'environ 170 ans, puisque datée de 1740 , elle est la plus proche dans le temps de celle de Liçarrague. Ainsi peut-on mettre en évidence que dès le $18^{\circ}$ ces morphèmes semblent avoir disparu de la langue, le cas de ai-, cependant, méritant une examen particulier de ce point de vue. 


\section{NOTE}

1. Tcstu hau Donostiako udako ikastaroetan emanikako hilzaldi bati dagokio. Gaia : Euskararen hisIoria : arazo zahar. bide berri (1997/07/30ctik 1997/08/01 arte).

2. Beste baldintza da adiz ergatibodunetan absolutiboa lehen edo bigarren pertsonakoa ez izatea. cuskalki gehienctan bederen : beza edo bitza vs nazala eta zaitzala.

3. Joan den mendearen ondarrean, bai van Eysek, bai Dogsonek, Schuchardtek eta Vinsonck cre nahi ukan zuten Leizarragaren eredu nagusia aurkitu, eta eztabaida biziak ere izan zituzten beren artcan grckozko. latinezko eta frantsesczko testuak zenbatetaraino baliatuak izan ziren izartzeko. Ruiz Arzallusek (1991) cztabaida horick gogorarazi ondoan, crakustera cmaten du, hipotesi arrazoizkoena dela Leizarraga frantses bertsio baten arabera ari izana zela. Van Eysek ere bere denboran agerian utzi zuen bezala, frantsesezko anitz bertsio argitararatu ziren eta gehientsuenak galdu izan baitira, arras neke da, edo ezinezkoa, Leizarragaren frantsesezko iturni haren aurkitzea.

4. 16. mendean, ahal morfema iraganari ez dagokion egnera bati bunuz baliatzen denean ez da izan cdo *edun aditz laguntzaileekin agertzen, baina bcti adizkera trinkockin, edo *edin cta *ezan (eta *iro) laguntzaileckin. Hots, ez dugu kausitzen, egun guziz arruntak izanagatik, egiten ahal dut bezalako formarik Etxcparc cta Lcizarragaren testuetan. Alderdi horretarik alba- aurrizkiarekin gauza bera gertatzea guziz normala da.

5. Oihenartck 384. efrefrauan albai(t)- forma erabili zuen : Otsoa lagun duanean, albaihu hora saihetsean.

6. Hemen dakargun adibidea (20) eztahaida baten sorburua gertatu da cuskal filologian. Ikus daitckecnaz, Lcizarragak dioena eta Haranederrek dioena elkarren aurkakoak dira. Lehenbizikoak delako etxe hartarik jalgitzeko manatzen du (handic ilki albeitzindeże), bigarrenak, berriz, ez ateratzeko dio (ez çaileżlela ilkhi handic). Desberdintasun horren iturburua grekozko testuaren eta Vulgataren arteko diferentzietan aurkitzen da. Leizarragak dioena grekozko testuari darraio, Haranederren interpretazioa, aldiz, Vulgataren crranari dagokio. Michelenak (1978) hori ikusiz pentsatu zuen menturaz Leizarragak grekozko testua batzuetan bchintzat segitzen zuela, Lafonek ere (1943) erran zuen bezala. Ordean, kasu honek alderdi horrctarik cz du gauza handirik frogatzen, baldin eta frantsesezkn bertsioan ere baiczkoan bada agindua. Ruiz Arzallusck (1991) 1561 cko bertsio baten lekukotasuna ekarri zuen gisa horretakoa, eta hemen dakarguna cre hajakoa da : \& vous en allez de ld. Beraz, Leizarragaren itzulpena hemen ere arras hurbiletik jarraikitzen zaio frantses testuari.

7. Hona lau adibideak:

- Egundan' ez ailiz jaio gaxiagina/Edertza. neskato onaren zegina./Edo jai'-eta, berhala/Hil ailiz, inhar bezala Oihenart. Neurnizak. 6, 13-16.

- Eta gaixtoencko kontuan, aillekitua hek cre. edo hek bedere geldi. Axular \$116

- Haur dira. (eta lainkoak ailliotsa liren asko) haragiaren bekhatuan ez eronzeko eta eroriz gero ere iaikitzcko hartu behar diren erremediorik prinzipalenak. Axular $\$ 271$.

8. Galda daitcke Pouvreauren lekukotasuna kasu honetan arras fidagarria ote den, ezen ai- batzuetan usic gabcko maneran crabili zucn, hala nola orainaldian - $n$ edo -la atzizkia zuten subjonktiboko formetan, cta pertsona marka edo denbora marka aurriki gisa zekarten formetan. Ondoko hiru adibideek hori erakusten digutc.

- ainadilla bada bizi egin merezi eta behar bezala. (lesusen Imitacionea. 203).

- Jesus Jauna erran eta prometalu duiun bezala. egin ere bedi hala, eta ainadin merezidun gertha. (Iesusen Imitacionea. p. 228)

- Berdin pairatu behar dut, eta aidezadan enteki paira, ekaitza eta tenpesta ioan dadin eta hobeki gerta dakidan artean. (Iesusen Initacionea. p. 170).

Ohargarri da lehen adibidcari dagokion pasartea Inchauspek ainendi erabiliz izzuli zuela :

Haur naiçula. çoure cerbutzaria. pres nuçu orotara : czpeiniz bici enetaco. bena bai çouretaco: ainendi ounxa eta çuc merechi bef̧ala! (Inchausperen itzulp. 154. o.)

Pouvrcau dakigun bezala cuskaldun berria zen. Berak bere buruz. analogiaz-edo, asmalu ote zituen forma horick ? Edo ai interjckzioa dugu hotrclakoetan, loturik idatzia ?Hots, ez aurrizkia, baina bestc moricma bal. 